\title{
Enhanced X-ray absorption by using gold nanoparticles in a biological tissue
}

\author{
A. Berrezoug, A.S.A Dib ${ }^{\star}$ and A.H. Belbachir \\ Laboratory of Analysis and Application of Radiation, Department of genie physics, University of Sciences and Technology M. Boudiaf, \\ B.P. 1505, Oran, Algeria.
}

Received 2 May 2015 - Accepted 24 August 2015

\begin{abstract}
In recent years, application of nanoparticles (NPs) in diagnosis and treatment of cancer has been the issue of extensive research. In this study, we investigated the effect of gold nanoparticles (GNPs) in a tumor during X-ray therapy. Our simulation, based on the Monte Carlo method, shows that the GNPs injected into a tumor considerably enhanced the absorbed dose during X-ray therapy, especially in the energy range between $10 \mathrm{keV}$ and $150 \mathrm{keV}$. This increase in the absorbed dose is due to a combination of increased photoelectric interaction and Auger electron generation from the gold atoms. Furthermore, the absorbed dose in a biological cell is strongly influenced by the size of the GNPs; our results show that the ideal diameter of GNPs should be around $50 \mathrm{~nm}$, and this result was confirmed by several authors.
\end{abstract}

Keywords: radiation dose / human organ / tumors / X-ray

\section{Introduction}

Radiation therapy is used in about $70 \%$ of all cancer treatments (Braunn et al., 2013); it allows killing tumor cells by depositing a high dose of radiation within the tumor. In radiotherapy, the photon beam may come from outside the body (external-beam radiation therapy), or it may come from radioactive material injected into the bloodstream or placed in the body near the tumor cells. Unfortunately, radiation therapy can damage normal cells as well as cancer cells. Therefore, to minimize side effects, the treatment must be carefully planned (Lawrence et al., 2008). The ultimate goal is to eradicate the disease without damaging the surrounding healthy tissues. Radiation therapy can directly damage the DNA or create charged particles inside a cell that can then damage the DNA.

Scientists are conducting research studies to learn how to use radiation therapy and treat cancer more effectively. Researchers are also studying radiosensitizers, radioprotectors, and especially nanomaterials that enhance a cell's response to radiation. Several agents are under study as radiosensitizers and many of them are currently being studied as potential radioprotectors (Connell and Hellman, 2009). With advances in the synthesis of a variety of nanomaterials, bio-nanomaterials have become an interesting subject in biomedical application (Xu et al., 2008; Porcel et al., 2010; Kim and Jon, 2012).

Nowadays, gold nanoparticles are emerging as promising agents for cancer therapy (Mesbahi, 2010;

\footnotetext{
^ dibanis70@gmail.com
}

McMahon et al., 2011; Jain et al., 2012; Tsiamas et al. 2013) ; they can be used as good material for diagnosis and treatment of cancer cells (Heath and Davis, 2008; Jiao et al., 2011). Numerous experimental and theoretical researchers are focusing on GNPs as a biomedical application because of their physical and chemical properties, and their biocompatibility (Giljohann et al., 2010; Misawa and Takahashi, 2011).

It is known that tumor cells are bigger than normal cells, and they have the capacity to consume more substances, therefore each tumor cell has the ability to contain more than one GNP; this depends essentially on the GNPs' size (Chow et al., 2012). However, it is useful to investigate the effect of GNPs on the absorption of X-ray beams at the cellular level.

\section{Methods and geometry}

Our main study is to investigate the effect of GNPs injected into a tumor during a diagnosis, or X-ray therapy, especially in the case of a tumor localized in a sensitive organ, such as the eye, or the head, etc. First, we simulated a spherical tumor localized in the center of a human head. Then, the human head is exposed to an X-ray placed $1 \mathrm{~m}$ from the patient (see Figure 1).

In the second part of this work, we were interested in studying the effect of GNPs on the cellular scale during X-ray exposure. For this, we simulated a spherical GNP in the center of a water cube with a volume of $20 \mu \mathrm{m}^{3}$. 


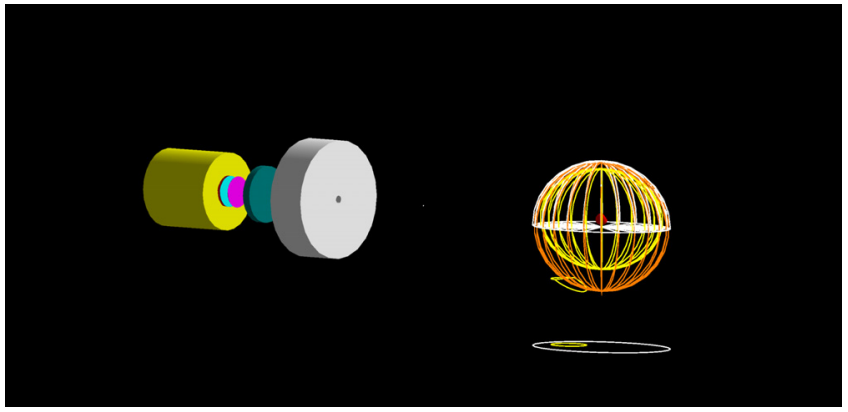

(a)

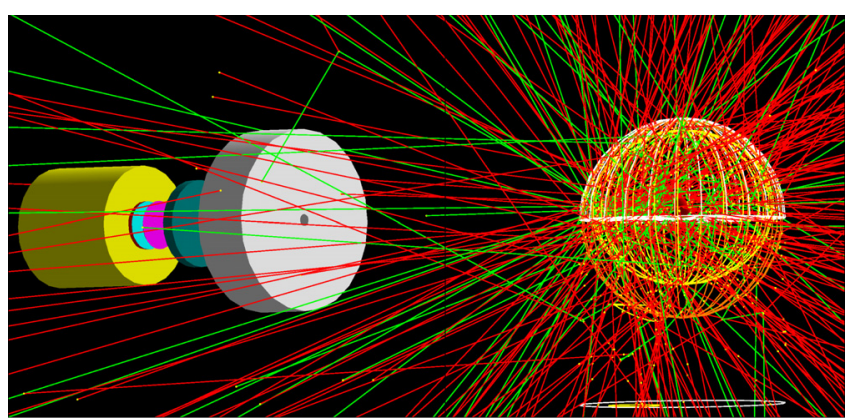

(b)

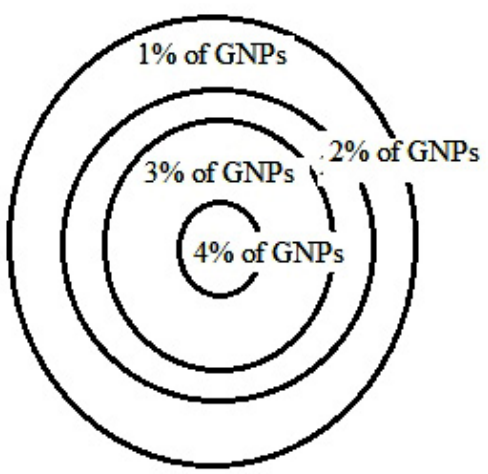

(c)

Figure 1. Materials and geometry: simulation of X-ray therapy of a tumor localized in the center of a human head. (a) Geometry of a human head: tumor localized in the center. (b) Geant 4 simulation of X-ray interaction with a human head. The green lines define secondary X-rays, and the red lines define secondary electrons. (c) Tumor geometry with different concentrations of GNPs inside: tumor with a spherical shape of a radius of $0.8 \mathrm{~cm}$.

\subsection{Monte Carlo simulations}

Our simulation is based on the Monte Carlo code Geant 4 (Agostinelli et al., 2003; Allison et al., 2006). Geant4 is a software toolkit for the simulation of the passage of particles through matter. It is used in various application domains (Chauvie et al., 2007), including high energy physics, astrophysics, space science, and medical physics.

This work is based on the Penelope physics model. The Penelope models have been specifically developed for Monte Carlo simulation and great care was given to the low energy description. Hence, these implementations provide reliable
Table 1. Physical processes of the Penelope physics model used in the Monte Carlo Code Geant4.

\begin{tabular}{lc}
\hline Particle & Physical processes \\
\hline Gamma (X-ray photon) & Photoelectric effect \\
& Compton Scattering \\
& by Linearly Polarized Gamma Rays \\
& Compton Scattering \\
& Rayleigh Scattering \\
& Multi-scattering effect \\
& Bremsstrahlung \\
Electron & Ionization \\
& Fluorescence \\
Atomic relaxation & Auger process \\
\hline
\end{tabular}

results for energies down to a few hundred $\mathrm{eV}$ and can be used up to $1 \mathrm{GeV}$. For this reason, they may be used in Geant 4 as an alternative to the Low Energy processes (Pandola et al., 2015). The physical processes used in this study are presented in Table 1.

\subsection{Materials and geometry}

\subsubsection{Simulation of a tumor inside a human head}

The X-ray therapy was simulated with the Monte Carlo code Geant 4 of a tumor within a human head. This tumor is assumed to have a spherical shape with a radius of $0.8 \mathrm{~cm}$ (see Figure 1c). Indeed, it is well known that the cells of a tumor tissue grow quickly; accordingly, they need more oxygenated blood than normal cells. Carmeliet and Jain (2000) studied the development of blood vessels in tumor cells in depth, and they noted that the blood vessels are more concentrated in the center of a tumor; this observation leads us to assume that the GNPs are distributed in the same way as the blood vessels in a tumor. Figure 1c shows the concentrations of GNPs localized in a tumor in our simulation. In this part of our simulation, we calculated the tumor absorbed dose during the X-ray exposure. Figure 2 shows the plot of the tumor absorbed dose both with and without GNPs versus X-ray energy. As can be seen, adding GNPs to a tumor considerably affects the tumor absorbed dose; this curve presents two peaks of energy around $50 \mathrm{keV}$ and $90 \mathrm{keV}$. We note here, for these two energy peaks, that the absorbed dose increases 4 to 5 times.

\subsubsection{GNP inside a biological Cell}

An individual GNP is embedded in a water volume that simulates a liquid tissue with sides of $20 \mu \mathrm{m}$. One face of this volume is uniformly irradiated with the X-ray spectrum under investigation. This GNP has a diameter ranging between $10 \mathrm{~nm}$ and $150 \mathrm{~nm}$, in order to investigate the effect of the GNP at a cellular level. We calculated the absorbed energy by a cell containing one GNP irradiated with a monochromatic $\mathrm{X}$-ray energy ranging between $10 \mathrm{keV}$ and $100 \mathrm{keV}$. From our results, we noted that the absorbed energy rises where the GNP is localized. With this simulation, we were interested in evaluating the absorbed energy of the GNP. We plotted the curve 


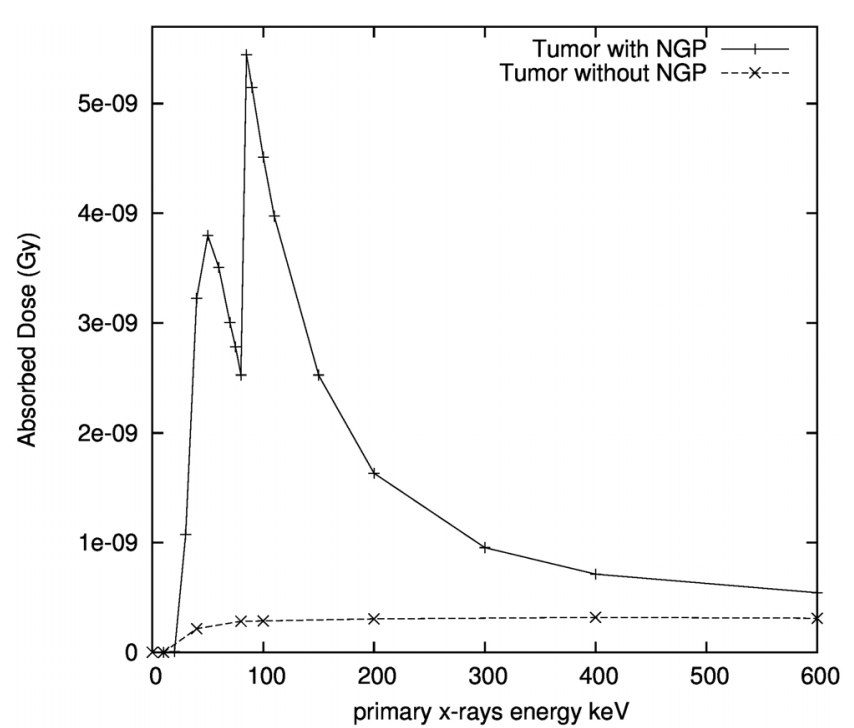

Figure 2. The absorbed dose versus $\mathrm{X}$-ray energy: case of a tumor localized in the brain.

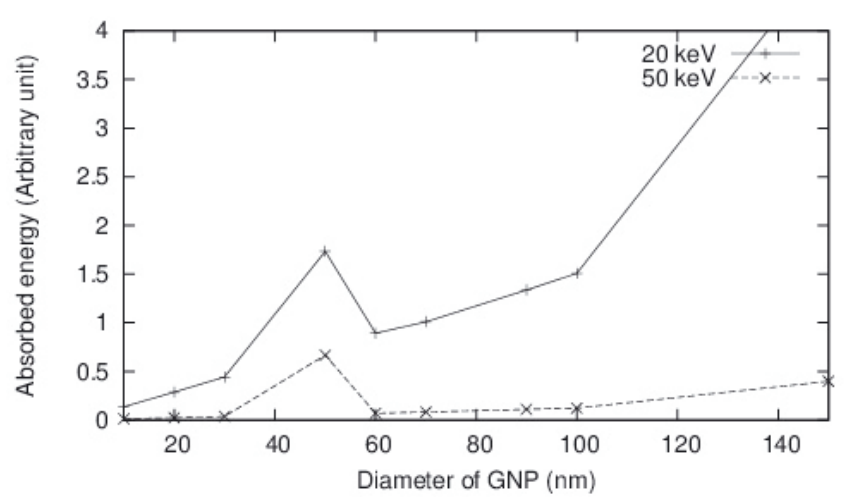

Figure 3. Energy absorbed by a biological cell versus the diameter of the GNP.

of the absorbed energy in relation to the size of the GNP (see Figure 3 ). This figure shows that the absorbed energy presents a peak at $50 \mathrm{~nm}$ in the interval ranging between 0 and $100 \mathrm{~nm}$. This result was confirmed by several authors (Jiang et al., 2008; Chithrani et al., 2010; Jain et al., 2012). It should be noted here that contrary to what was expected, the absorbed energy for an X-ray energy of $20 \mathrm{keV}$ is higher than that of an energy of $50 \mathrm{keV}$.

On the other hand, we calculated the absorbed energy in a tissue containing a GNP of $50 \mathrm{~nm}$ irradiated by an X-ray energy ranging between $10 \mathrm{keV}$ and $100 \mathrm{keV}$. Figure 4 shows a plot of the absorbed energy versus X-ray energy. We note the existence of three peaks at energies of $20 \mathrm{keV}, 50 \mathrm{keV}$ and $90 \mathrm{keV}$. The first energy peak is the highest; almost twice the second energy peak and four times the fourth energy peak.

We note here, that for a GNP of $50 \mathrm{~nm}$ in diameter, the $\mathrm{X}$-ray energy of $20 \mathrm{keV}$ is the most favorable in order to increase the absorption energy in a biological cell. These results presented in Figure 4 are not in contradiction with the previous results shown in Figure 2. The absorbed dose represents the mean energy imparted to matter per unit mass by ionizing

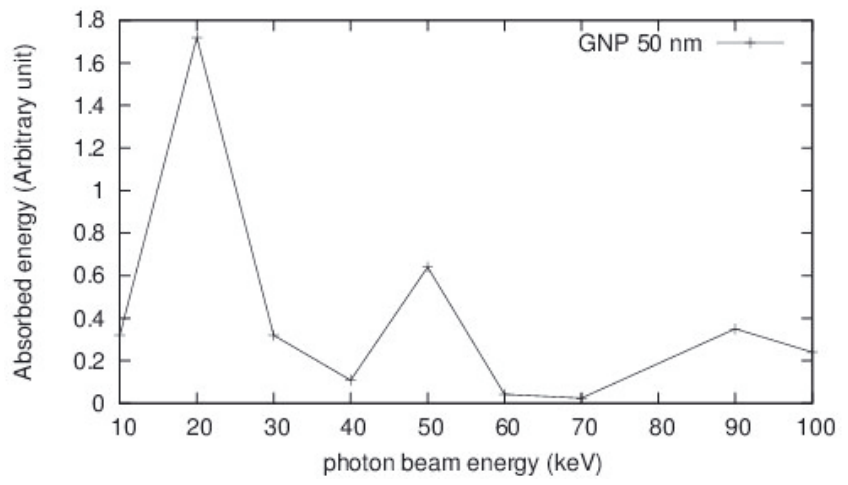

Figure 4. Energy absorbed by a biological cell within a GNP with a size of $50 \mathrm{~nm}$ versus photon beam energy.

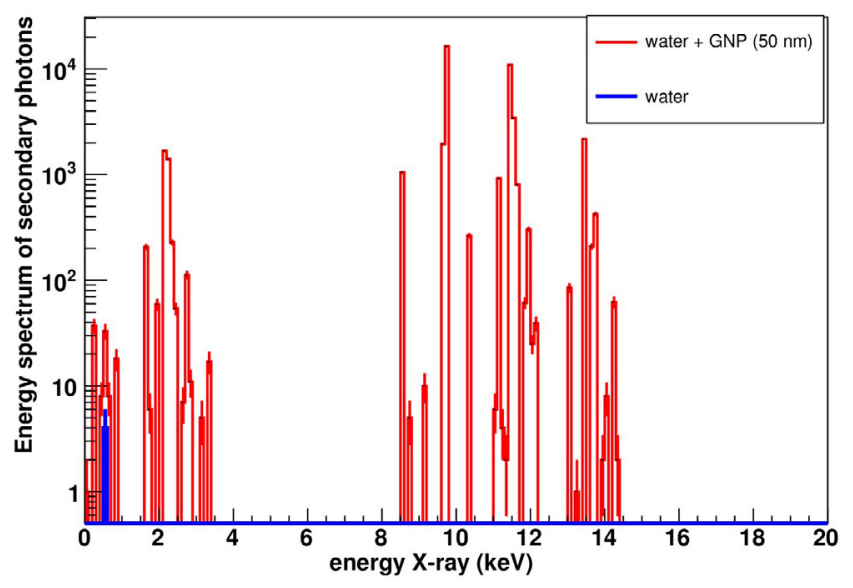

Figure 5. Energy spectrum of secondary photons from an X-ray energy of $20 \mathrm{keV}$ with a liquid tissue (GNP size of $50 \mathrm{~nm}$ ).

radiation. This explains the disappearance of the energy peak at $20 \mathrm{keV}$ in Figure 2.

\subsection{Secondary particles}

In this section, we were interested in evaluating the secondary particles created during X-ray irradiation from a GNP within a biological cell. These secondary particles, which contribute significantly to dose deposition and GNP ionization rates, are generated from the physical processes mentioned in Table 1. From the previous results, the absorbed energy is the highest when an X-ray energy of $20 \mathrm{keV}$ interacts with a biological cell containing a GNP of $50 \mathrm{~nm}$ in diameter. In this energy range, most of the secondary electrons and photons are emitted from the photoelectric effect, Auger effect and ionization effect.

\subsubsection{Energy spectrum of secondary photons}

The energy spectrum of the emitted photons from the interaction of an X-ray energy of $20 \mathrm{keV}$ with a biological cell containing a GNP is shown in Figure 5. As this figure shows, 


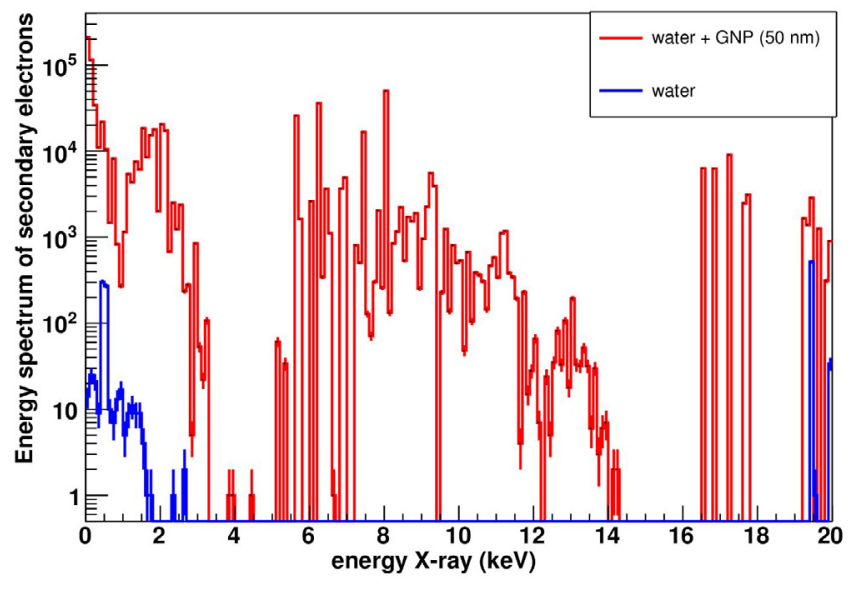

Figure 6. Energy spectrum of secondary electrons from an X-ray energy of $20 \mathrm{keV}$ with a liquid tissue (GNP size of $50 \mathrm{~nm}$ ).

gold atoms in a biological cell considerably improve the creation of secondary photons; these secondary photons are created from gold atomic relaxation. These results confirm that GNPs in a tissue can be used as radiosensitizers (Cheong et al., 2010; Kominami et al., 2011; Ricketts et al., 2012). Moreover, these photons created inside a biological cell contribute to depositing their energies in the area to be treated, and that will help to kill cancer cells.

\subsubsection{Energy spectrum of secondary electrons}

In our case, the GNP enhances the creation of secondary electrons; these electrons can be produced from the photoelectric effect, Auger effect and ionization effect. Figure 6 shows the energy spectrum of secondary electrons from the interaction of an X-ray energy of $20 \mathrm{keV}$ with a GNP within a biological cell. By comparing this figure with Figure 5, the energy spectrum of Auger electrons is between $8 \mathrm{keV}$ and $15 \mathrm{keV}$. This large number of low-energy electrons can cause serious damage to cancer cells and stop cell division: this is a new form of therapy, known as Auger therapy.

\section{Discussion and conclusion}

Most researchers are interested in modifying the existing drugs to improve their pharmacokinetics. Jiang et al. (2008) synthesized GNPs of controlled sizes ranging between $2 \mathrm{~nm}$ and $100 \mathrm{~nm}$; they noted that the optimal size for GNPs in a biological cell was between $40 \mathrm{~nm}$ and $50 \mathrm{~nm}$.

Moreover, Zhang et al. (2012) investigated the sizedependent radio-sensitization of GNPs for cancer radiation therapy in vitro and in vivo. They noted a greater sensitization effect for GNPs of $12.1 \mathrm{~nm}$ and $27.3 \mathrm{~nm}$ of than $4.8 \mathrm{~nm}$ and $46.6 \mathrm{~nm}$ particles. On the other hand, Chow et al. (2012) investigated the low-energy electrons (LEEs) produced when a GNP is irradiated by photon beams. It was found that the energy distribution of LEEs from GNPs does not vary significantly between different photon beam energies.
Based on the Monte Carlo code Geant4, in this paper we described the application of GNPs in X-ray therapy of cancerous cells. First, we simulated a spherical tumor localized in the center of a human head. In the second part of this work, we were interested in the effect of a GNP included in a biological cell during X-ray therapy.

Our results show that radiation therapy of cancerous cells in the presence of GNPs is more effective than pure irradiation (Figure 2). In fact, gold atoms increase the production of secondary electrons and photons in cancerous cells; these lowenergy electrons cause serious damage to cancer cells and stop cell division. From our simulation results, we conclude that in X-ray therapy, the best GNP size that should be used is around $50 \mathrm{~nm}$ in diameter, and this result was confirmed by several authors. In addition, to enhance the dose absorbed by a tumor, suitable X-ray energy should be used in the energy range between $20 \mathrm{keV}$ and $150 \mathrm{keV}$.

\section{References}

Agostinelli S. et al. (2003) Geant4 - a simulation toolkit, Nucl. Instrum. Meth. A 506, 250-303.

Allison J. et al. (2006) Geant4 developments and applications, IEEE Trans. Nucl. Sci. 53 (1), 270-278.

Braunn B., Boudard Colin A.J., Cugnon J., Cussol D., David J.C., Kaitaniemi P., Labalme M., Leray S., Mancusi D. (2013) Comparisons of hadrontherapy-relevant data to nuclear interaction codes in the Geant4 toolkit, J. Phys.: Conf. Ser. 420, 012163.

Carmeliet P., Jain R.K. (2000) Angiogenesis in cancer and other diseases, Nature 407 (6801), 249-257.

Chauvie S. et al. (2007) Geant4 physics processes for microdosimetry simulation: design foundation and implementation of the first set of models, IEEE Trans. Nucl. Sci. 54, 261928.

Cheong S.-K., Jones B.L., Siddiqi A.K., Liu F., Manohar N., Cho S.H. (2010) X-ray fluorescence computed tomography (XFCT) imaging of gold nanoparticle-loaded objects using $110 \mathrm{kVp}$ X-rays, Phys. Med. Biol. 55, 647-662.

Chithrani D.B., Jelveh S., Jalali F., Prooijen M.V., Allen C., Bristow R.G., Hill R.P., Jaffray D.A. (2010) Gold Nanoparticles as Radiation Sensitizers in Cancer Therapy, Radiat. Res. 173 (6), 719-728.

Chow J.C.L., Leung M.K.K., Jaffray D.A. (2012) Monte Carlo simulation on a gold nanoparticle irradiated by electron beams, Phys. Med. Biol. 57, 3323-3331.

Connell P., Hellman S. (2009) Advances in radiotherapy and implications for the next century: A historical perspective, Cancer Res. 69 (2), 383-392.

Giljohann D.A., Seferos D.S., Daniel W.L., Massich M.D., Patel P.C., Mirkin C.A. (2010) Gold nanoparticles for biology and medicine, Angew. Chem. Int. Ed. 49 (19), 3280-3294.

Heath J.R., Davis M.E. (2008) Nanotechnology and cancer, Annu. Rev. Med. 59 (1), 251-265.

Jain S., Hirst D.G., O’Sullivan J.M. (2012) Gold Nanoparticle as novel agents for cancer therapy, Br. J. Radiol. 85, 101-113.

Jiang W., Kim B.Y.S., Rutka J.T., Chan W.C.W. (2008) Nanoparticlemediated cellular response is size-dependent, Nat. Nanotechnol. 3, 145-150.

Jiao P.F., Zhou H.Y., Chen L.X., Yan B. (2011) Cancer-targeting multifunctionalized gold nanoparticles in imaging and therapy, Current Medicinal Chemistry 18, 2086-2102. 
Kim D., Jon S. (2012) Gold nanoparticles in image-guided cancer therapy, Inorganica Chimica Acta 393, 154-164.

Kominami H., Tanaka A., Hashimoto K. (2011) Gold nanoparticles supported on cerium (IV) oxide powder for mineralization of organic acids in aqueous suspensions under irradiation of visible light of $\lambda=530 \mathrm{~nm}$, Applied Catalysis A 397, 121-126.

Lawrence T.S., Ten Haken R.K., Giaccia A. (2008) Principles of Radiation Oncology. In: Cancer: Principles and Practice of Oncology, 8th ed. (V.T. DeVita Jr., T.S. Lawrence, S.A. Rosenberg, Eds.). Lippincott Williams and Wilkins, Philadelphia.

McMahon S.J. et al. (2011) Nanodosimetric Effects of Gold Nanoparticles in Megavoltage Radiation Therapy, Radiother. Oncol. 100, 412-416.

Mesbahi A. (2010) A review on gold nanoparticles radiosensitization effect in radiation therapy of cancer, Reports of practical oncology and radiotherapy, 15, 176-180.

Misawa M., Takahashi J. (2011) Generation of reactive oxygen species induced by gold nanoparticles under X-ray and UV irradiations, Nanomedicine: Nanotechnology, Biology, and Medicine 7, 604-614.
Pandola L. et al. (2015) Validation of the Geant4 simulation of bremsstrahlung from thick targets below $3 \mathrm{MeV}$, Nucl. Instrum. Methods Phys. Res. B 350, 41-48.

Porcel E. et al. (2010) Platinum nanoparticles: a promising material for future cancer therapy? Nanotechnology 21, 085-103.

Ricketts K., Castoldi A., Guazzoni C., Ozkan C., Christodoulou C., Gibson A.P., Royle G.J. (2012) A quantitative X-ray detection system for gold nanoparticle tumour biomarkers, Phys. Med. Biol. 57, 5543-5555.

Tsiamas P. et al. (2013) Impact of beam quality on megavoltage radiotherapy treatment techniques utilizing gold nanoparticles for dose enhancement, Phys. Med. Biol. 58, 451-464.

Xu Y.J. et al. (2008) Dosimetric Analyses of Single Particle Microbeam in Cell Irradiation Experiment, Plasma Sci. Technol. 10 (6), 764-768.

Zhang X.D., Wu D., Shen X., Chen J., Sun Y.M., Lu P.X., Liang X.J. (2012) Size-dependent radiosensitization of PEG-coated Gold nanoparticles for cancer radiation therapy, Biomaterials 33, 6408-6419.

Cite this article as: A. Berrezoug, A.S.A Dib, A.H. Belbachir . Enhanced X-ray absorption by using gold nanoparticles in a biological tissue. Radioprotection 50(4), 281-285 (2015). 